OPEN ACCESS

Edited by:

David Kadosh

The University of Texas Health Science Center at San Antonio, United States

Reviewed by:

Derek Thomas,

Grand Valley State University,

United States

Vishukumar Aimanianda

Institut Pasteur, France

${ }^{*}$ Correspondence:

Markus Kostrzewa

markus.kostrzewa@bruker.com

Katrin Sparbier

katrin.sparbier@bruker.com

Specialty section:

This article was submitted to

Fungal Pathogenesis,

a section of the journal

Frontiers in Cellular and Infection

Microbiology

Received: 26 October 2018

Accepted: 22 January 2019

Published: 18 February 2019

Citation:

Vatanshenassan M, Boekhout T, Meis JF, Berman J, Chowdhary A,

Ben-Ami R, Sparbier $K$ and Kostrzewa M (2019) Candida auris Identification and Rapid Antifungal

Susceptibility Testing Against Echinocandins by MALDI-TOFMS.

Front. Cell. Infect. Microbiol. 9:20.

doi: 10.3389/fcimb.2019.00020

\section{Candida auris Identification and Rapid Antifungal Susceptibility Testing Against Echinocandins by MALDI-TOF MS}

\author{
Mansoureh Vatanshenassan ${ }^{1,2}$, Teun Boekhout ${ }^{2,3}$, Jacques F. Meis ${ }^{4,5}$, Judith Berman ${ }^{6}$, \\ Anuradha Chowdhary ${ }^{7}$, Ronen Ben-Ami ${ }^{8,9}$, Katrin Sparbier ${ }^{1 *}$ and Markus Kostrzewa ${ }^{1 *}$ \\ ${ }^{1}$ Bruker Daltonik GmbH, Bremen, Germany, ${ }^{2}$ Institute for Biodiversity and Ecosystem Dynamics, University of Amsterdam, \\ Amsterdam, Netherlands, ${ }^{3}$ Westerdijk Fungal Biodiversity Institute, Basidiomycete and Yeast Research, Utrecht, \\ Netherlands, ${ }^{4}$ Department of Medical Microbiology and Infectious Diseases, Canisius Wilhelmina Hospital, Nijmegen, \\ Netherlands, ${ }^{5}$ Centre of Expertise in Mycology Radboudumc, CWZ, Nijmegen, Netherlands, ${ }^{6}$ Department of Molecular \\ Microbiology and Biotechnology, George S. Wise Faculty of Life Sciences, Tel Aviv University, Tel Aviv, Israel, ${ }^{7}$ Department of \\ Medical Mycology, Vallabhbhai Patel Chest Institute, University of Delhi, New Delhi, India, ${ }^{8}$ Infectious Diseases Unit, Tel Aviv \\ Sourasky Medical Center, Tel Aviv, Israel, ${ }^{9}$ Sackler Faculty of Medicine, Tel Aviv University, Tel Aviv, Israel
}

Candida auris was first reported in an ear swab from Japan in 2009; it then promptly spread over five continents and turned into a global nosocomial problem. The main challenges faced by many researchers are the mis-identification by conventional methods in clinical laboratories and failure in treatment. About $90 \%$ of C. auris strains are intrinsically resistant to fluconazole (FLU), and it is developing resistance to multiple classes of available antifungals. Echinocandins are the most potent class of antifungals against $C$. auris; however, reduced susceptibility to one or many echinocandin drugs has been recently observed. Thus, the main issues addressed in this paper are the fast and accurate identification of $C$. auris derived from Sabouraud dextrose agar and blood culture bottles as well as the rapid antifungal susceptibility test by MALDI-TOF MS. This study successfully identified all isolates of $C$. auris $(n=50)$ by MALDI-TOF MS, with an average log score of $\geq 2$. An accuracy of $100 \%$ was found on both agar plate and blood culture bottles. MALDI Biotyper antibiotic susceptibility test-rapid assay (MBT ASTRA) was used for rapid antifungal susceptibility testing (AFST). A comparison between MBT ASTRA and the Clinical and Laboratory Standards Institute guidelines (CLSI) detected a sensitivity and specificity of $100 \%$ and $98 \%$ for anidulafungin, and $100 \%$ and $95.5 \%$ for micafungin, respectively. A categorical agreement of $98 \%$ and $96 \%$ was calculated for the two methods. For caspofungin, sensitivity and specificity of 100 and $73 \%$ were found, respectively, with a categorical agreement of $82 \%$. MBT ASTRA has the great potential to detect $C$. auris isolates non-susceptible against echinocandin antifungals within $6 \mathrm{~h}$, which makes it a promising candidate for AFST in clinical laboratories in the future.

Keywords: C. auris, MALDI-TOF MS, Identification, rapid antifungal susceptibility testing, echinocandins 


\section{INTRODUCTION}

Candida auris is a recently-emerged Candida species first isolated from human samples of the external ear in Japan in 2009 (Satoh et al., 2009), and had spread to more than 30 countries in $<10$ years (Saris et al., 2018). C. auris is primarily detected in patients with a long period of hospitalization in intensive care units (ICU). It causes diseases ranging from superficial skin infections to invasive bloodstream infections (BSI) with high mortality rates (30\% to 60\%) (Vincent et al., 2009; Chowdhary et al., 2016, 2017; Prakash et al., 2016; Cortegiani et al., 2018; Ruiz-Gaitán et al., 2018). In some hospitals in Asia C. auris is the second most common isolated species from blood cultures (Mathur et al., 2018). C. auris is resistant to fluconazole (FLU), and is also regularly reported as a multidrug resistant (MDR) yeast (Lee et al., 2011; Cortegiani et al., 2018; Meis and Chowdhary, 2018). Previous published studies showed increased C. auris MICs to all three major antifungal classes (Shin et al., 2012; Arendrup and Patterson, 2017; Lockhart et al., 2017; Chowdhary et al., 2018; Cortegiani et al., 2018). Echinocandins are the most effective drugs against C. auris infections (Sears and Schwartz, 2017; Jeffery-Smith et al., 2018). The majority of studies have proposed a tentative MIC breakpoint (in $\mu \mathrm{g} / \mathrm{mL}$ ) for resistance i.e., $\geq 4$ for anidulafungin and micafungin, and a MIC breakpoint $\geq 2$ for caspofungin (Ben-Ami et al., 2017; Spivak and Hanson, 2018; Tsay et al., 2018). In addition, echinocandin-resistant $C$. auris isolates have been observed on rare occasions in different geographical areas (Chowdhary et al., 2018). Notably, 36\% of echinocandin resistant Candida strains have cross-resistance to azole antifungals (Arendrup and Patterson, 2017; Arendrup et al., 2017b; Chowdhary et al., 2018). Thus, knowing the degree of echinocandin resistance in specific C. auris isolates is critical for choosing appropriate antifungal drug therapeutic strategies. Considering the MDR propensity of this yeast the treatment of $C$. auris remains a challenge as also its identification in the routine microbiology laboratories (Ben-Ami et al., 2017; Spivak and Hanson, 2018). C. auris has been misidentified as C. haemulonii, C. famata, and even $S$. cerevisiae by conventional biochemical techniques and some commercial methods (Chowdhary et al., 2013; Ceyssens et al., 2017; Spivak and Hanson, 2018). Several studies have reported misidentification of $C$. auris as $C$. haemulonii by VITEK 2, if the database is not updated (Girard et al., 2016; Ben-Ami et al., 2017; Khan et al., 2018). Molecular methods remain the most reliable and accurate available approaches, although they are expensive and not used routinely in clinical laboratories (Ben-Ami et al., 2017; Spivak and Hanson, 2018; Tsay et al., 2018). Matrix-assisted laser desorption ionization-time of flight mass spectrometry (MALDI-TOF MS) has recently been considered as a convenient, rapid and high throughput technology in the identification of variant microorganisms at the species level (Cendejas-Bueno et al., 2012; Croxatto et al., 2012; Kolecka et al., 2016). Some studies showed that MALDI-TOF MS can identify C. auris correctly and rapidly, differentiating it from other related species (Ghosh et al., 2015; Kathuria et al., 2015; Prakash et al., 2016; Schelenz et al., 2016). However, the specificity and sensitivity of MALDI-TOF MS to a collection of $C$. auris isolates has not been tested. Taken together, identification and antifungal susceptibility tests for $C$. auris infection remain a challenge in the clinical microbiology setting.

Here, we investigated the MALDI-TOF MS based identification of $C$. auris. In addition, since a new trend to apply MALDI-TOF MS for AFST has appeared, Vella et al. (2013) have developed a rapid new AFST assay based on MALDI-TOF MS (Vella et al., 2017). They analyzed changes in the MS profile spectra induced by antifungals after $3 \mathrm{~h}$ of incubation (Vella et al., 2013). Although this method successfully worked for C. albicans isolates resistant to caspofungin (Vella et al., 2013), it did not accurately work for C. glabrata strains resistant to anidulafungin with known FKS2 mutations (Vella et al., 2017). Therefore, more recently, MALDI Biotyper antibiotic susceptibility test- rapid assay (MBT ASTRA) was introduced for the rapid detection of C. albicans and C. glabrata strains resistant against caspofungin (Vatanshenassan et al., 2018), where resistant strains were detected within $6 \mathrm{~h}$ with a high sensitivity and specificity for both species. This method is a semi-quantitative phenotypic assay based on the comparison of cell growth in the presence of an antifungal to growth in a control setup without antifungal drug. The present research explores, for the first time, the capability of the MBT ASTRA to detect non-susceptible $C$. auris against the echinocandin drug class, and the degree to which they agree with results from CLSI microdilution assays.

\section{MATERIALS AND METHODS}

\section{Candida auris Isolates}

The isolates used in this study were obtained from either strain collections or stock cultures of routine samples for which no written informed consent is required. A total of 50 C. auris isolates derived from either strain collections (10 from the Centers for Disease Control and Prevention, United States and 23 isolates from the Westerdijk Institute, The Netherlands) or stock cultures of routine samples (17 isolates) of different geographical areas (India, Israel, South Africa) were analyzed (Supplementary Table 1). All isolates had already been identified as C. auris by different commercial and molecular techniques. The isolates were stored at $-80^{\circ} \mathrm{C}$ and fresh overnight cultures on Sabouraud dextrose agar (SDA) were used for identification and antifungal susceptibility tests (AFST). The reference strains C. parapsilosis ATCC 22019 and C. krusei (Pichia kudriavzevii) ATCC 6258 , were used as quality control strains for susceptibility testing by the CLSI microdilution method. Two C. auris strains, CBS 12372 (KCTC 17809) and CBS 10913 (DSM 21092), were used as quality control strains for echinocandin susceptibility testing by the CLSI method and the MBT ASTRA (Arendrup et al., 2017a; Ruiz-Gaitán et al., 2018).

\section{MALDI-TOF MS Species Identification}

For the MALDI-TOF MS-based identification of C. auris the Bruker MBT Compass Library, Revision E MBT 7854 MSP Library was employed. Samples derived from agar plate were 
prepared according to the MALDI Biotyper standard protocol (Sauer et al., 2008; Clark et al., 2013; Vlek et al., 2014).

For species identification from blood cultures, blood culture bottles (BD Bactec Plus Aerobic/F; Becton Dickinson, Heidelberg, Germany) were enriched with $10 \mathrm{ml}$ whole sheep blood and spiked with the respective $C$. auris strains. The bottles were incubated in a Bactec automated blood culture instrument (Becton Dickinson, Heidelberg, Germany) until they were positive for each isolate. Subsequently, the positive blood cultures were purified by MALDI Sepsityper kit (Bruker Daltonik GmbH, Germany) according to the manufacturer's recommendations (Clark et al., 2013; Lange et al., 2014; Vlek et al., 2014).

\section{CLSI Microbroth Susceptibility Testing}

The MICs for anidulafungin (Pfizer, New York, United States), micafungin (Astellas, Toyama, Japan) and caspofungin (SigmaAldrich, Germany) were determined in duplicate by the CLSI standard microdilution method according to guideline M60 (November 2017) (Clinical Laboratory Standards Institute. Performance Standards for Antifungal Susceptibility Testing of Yeasts; approved, 2017). Slow-growing strains that could not be visually evaluated after $24 \mathrm{~h}$, were analyzed after $48 \mathrm{~h}$ incubation at $37^{\circ} \mathrm{C}$. So far, respective breakpoints for susceptibility/resistance classification have not been defined by CLSI and are suggested in this study.

\section{MBT ASTRA}

The in vitro antifungal susceptibility test was carried out according to the standard MBT ASTRA method, as recently described (Vatanshenassan et al., 2018), using 2 fold serial dilutions of anidulafungin (ranging from 0.125 to $8 \mu \mathrm{g} / \mathrm{ml}$ ), micafungin (ranging from 0.5 to $32 \mu \mathrm{g} / \mathrm{ml}$ ), and caspofungin (ranging from 0.125 to $4 \mu \mathrm{g} / \mathrm{ml}$ ) and an additional control without an antifungal. Briefly, supplemented RPMI 1640 medium (Sigma-Aldrich, Germany) and cell inoculum were prepared according to the EUCAST reference method (Arendrup et al., 2017a; Vatanshenassan et al., 2018). Incubation was performed at $37^{\circ} \mathrm{C}$ in a Thermo Mixer (Eppendorf, Germany) under agitation at $300 \mathrm{rpm}$ for $6 \mathrm{~h}$. Multi-well filter plates ( $1 \mathrm{ml}$ well, $0.45 \mu \mathrm{m}$ GHP, PALL, United States) were used to collect the cells after incubation by centrifugation at $4,000 \times \mathrm{g}$ for $5 \mathrm{~min}$ (Eppendorf, Germany). Next, the cells were rinsed twice with $200 \mu \mathrm{l}$ sterile deionized water and once with $100 \mu \mathrm{l} 75 \%$ ethanol. Cell lysis was performed by $10.5 \mu 170 \%$ formic acid (Merck, Germany) and $10.5 \mu \mathrm{l}$ 100\% acetonitrile (Roth, Germany) directly on the filter and was repeated once again. For MALDI-TOF MS measurements, $1 \mu l$ lysate of each set up was spotted in duplicate onto a polished steel target plate (Bruker Daltonik, Germany) and overlaid with $1 \mu \mathrm{l}$ MALDI matrix $(10 \mathrm{mg} / \mathrm{ml}$ of $\alpha$-cyano-4-hydroxy-cinnamic acid [ $\alpha$-HCCA $]$ in $50 \%$ acetonitrile-2.5\% trifluoroacetic acid; Bruker Daltonik, Germany) containing the MBT ASTRA Standard II (Bruker Daltonik, Germany). MALDI-TOF MS spectra were acquired and analyzed by MBT ASTRA prototype software (Cendejas-Bueno et al., 2012; Croxatto et al., 2012; Vatanshenassan et al., 2018).

\section{MBT ASTRA From Positive Blood Cultures}

Twenty isolates from the strains described above were randomly selected; susceptible $(n=14)$ and non- susceptible $(n=6)$ strains to anidulafungin and micafungin, susceptible $(n=13)$, or nonsusceptible $(n=7)$ to caspofungin. Preparation of blood cultures, incubation, and purification of positive cultures by MALDI Sepsityper kit was performed exactly as described above for strain identification. The pellet derived from the Sepsityper kit was resuspended in $1 \mathrm{ml}$ RPMI 1640 medium and used to prepare cell suspension of McF 0.5. All following steps of MBT ASTRA were performed as described above (Vatanshenassan et al., 2018).

\section{Data Analysis}

MBT ASTRA prototype software written in " $R$ " was employed for spectra analysis according to the procedure described by Lange et al. (Gibb and Strimmer, 2012; Lange et al., 2014; Vatanshenassan et al., 2018). First, the area under the curve (AUC) was calculated which is directly corresponding to the growth of the strain within the respective setup. Subsequently, the relative growth was calculated as a measure of the growth of the respective strain in the presence of antifungal: $R G=$ $\left(\mathrm{AUC}_{\mathrm{RPMI}+\text { antifungal }}\right) /\left(\mathrm{AUC}_{\mathrm{RPMI}}\right)$. The relative growth cut-off was set to $0.7 \mathrm{RG}$ units for all experiments. Strains with an RG value above this threshold were considered as non-susceptible; strain with an RG similar or below 0.7 were considered as susceptible. The CLSI microdilution was considered as standard method for evaluation of MBT ASTRA. Since this study was only a proof-of-principle-study, the number of tested strains was limited for applying a specific statistical calculation.

\section{RESULTS}

\section{Strain Identification}

All 50 strains of C. auris grown on SDA were correctly identified based on the MALDI Biotyper. The results, as shown in Table 1, indicate that 47 strains were identified with $\log$ (score) values of $\geq 2$ and three strains with $\log$ (score) values ranging between 1.7 and 2. Likewise, identification from positive blood culture bottles detected 46 strains with high $\log$ (score) values $\geq 2$ and only 4 strains with $\log$ (score) values ranging between 1.7 and 2. An absolute accuracy of $100 \%$ was calculated for C. auris identification using SDA and blood culture bottles (Table 1).

\section{CLSI Antifungal Susceptibility Test}

The CLSI microdilution method was considered as the gold standard to evaluate the MICs obtained by the MBT ASTRA. Since the CLSI has not yet determined the breakpoints for C. auris against echinocandins, the MICs found in this study based on CLSI microdilution method, were considered as a reference to evaluate breakpoints obtained by MBT ASTRA. Accordingly, strains were divided into 44 susceptible and 6 non-susceptible strains against anidulafungin and micafungin. All six isolates harbored an S639F mutation in FKS1 gene as published previously (Chowdhary et al., 2018). Applying the 
CLSI method for caspofungin divided strains into 33 susceptible and 17 non-susceptible isolates (Table 2). All 6 non-susceptible strains confirmed by sequencing, were correctly detected by CLSI method. As shown in Table 3, the MICs based on CLSI microdilution were defined as follows: $\mathrm{R}>4 \mu \mathrm{g} / \mathrm{ml}$ and $\mathrm{S} \leq$ $4 \mu \mathrm{g} / \mathrm{ml}$ for anidulafungin, $\mathrm{R}>8 \mu \mathrm{g} / \mathrm{ml}$ and $\mathrm{S} \leq 8 \mu \mathrm{g} / \mathrm{ml}$ for micafungin, and $\mathrm{R} \geq 1 \mu \mathrm{g} / \mathrm{ml}$ and $\mathrm{S}<1 \mu \mathrm{g} / \mathrm{ml}$ for caspofungin, respectively (Table 3) (Supplementary Table $\mathbf{1}$ ).

\section{In vitro Susceptibility Test by MBT ASTRA}

MBT ASTRA was performed for 50 strains as described above, and the area under the curve (AUC) was calculated by MBT ASTRA prototype software, based on the acquired spectra according to the growth of each strain at each antifungal concentration (Gibb and Strimmer, 2012). Further analysis determined that the minimal antifungal concentrations resulting in an RG value similar to or below the cut-off value of 0.7 were considered as the breakpoint for the respective strain. The RG values for four test strains (2 susceptible and 2 non-susceptible) are shown in Figure 1. For anidulafungin, the AUCs of the non-susceptible isolates were the same in absence and presence of the antifungal resulting in RGs close to 1; while for the susceptible strains, the AUCs were remarkably decreased in the presence of the drug resulting in decreasing RG values with increasing antifungal concentrations. The RG values of 42 susceptible strains already decreased at a concentration of $\leq 2 \mu \mathrm{g} / \mathrm{ml}$ of anidulafungin and for only one susceptible strain at a concentration of $4 \mu \mathrm{g} / \mathrm{ml}$. An example of the respective boxplot is given in Figure 1. Next, the MICs derived from MBT ASTRA were determined, and subsequently compared to the MICs derived from CLSI microdilution (Figure 2) (Table 3). Accordingly, $4 \mu \mathrm{g} / \mathrm{mL}$ was defined as the anidulafungin breakpoint concentration. Findings indicated that 49 of 50 strains were classified correctly, with only one susceptible strain mis-classified by MBT ASTRA.

For micafungin, susceptible strains had a growth reduction only at higher concentrations of micafungin compared to anidulafungin. For micafungin at a concentration of $8 \mu \mathrm{g} / \mathrm{mL}$, 42 susceptible strains were below the cut-off of 0.7 . In contrast, all non-susceptible strains constantly grew above the cut-off of 0.7 for all concentrations up to $32 \mu \mathrm{g} / \mathrm{ml}$. Table 3 shows that micafungin MBT ASTRA-MICs were in high agreement with the MICs derived from CLSI microdilution. Accordingly, there is a clear separation of susceptible and non-susceptible strains for micafungin with a breakpoint concentration of $8 \mu \mathrm{g} / \mathrm{ml}$ for both the CLSI reference method and MBT ASTRA. In addition, analysis of caspofungin susceptibility of the isolates found that the RGs of susceptible isolates were decreased at concentrations of $2 \mu \mathrm{g} / \mathrm{ml}$, while the growth for non-susceptible strains at this concentration was in the same range that had been observed for the controls for all caspofungin concentrations. The breakpoint for the MBT ASTRA was one serial dilution higher than the separating MIC for the CLSI method (Table 3).

Overall, analysis of all 50 tested isolates by MBT ASTRA indicated a high agreement between anidulafungin and micafungin. One susceptible isolate was mis-labeled by both antifungals, but all 6 strains determined as being non-susceptible by microdilution were correctly categorized by MBT ASTRA (Table 2). For anidulafungin, a high agreement of $98 \%$, and sensitivity and specificity of $100 \%$ and $98 \%$ were calculated, respectively. For micafungin, categorical agreement was 96\%, and sensitivity and specificity were $100 \%$ and $95.5 \%$, respectively (Table 4). In contrast, for caspofungin twenty-four susceptible and 26 non-susceptible isolates were detected by MBT ASTRA (Table 2). All 17 strains classified as non-susceptible by the CLSI method were determined also as non-susceptible by MBT ASTRA. However, 9 out of 33 strains that were categorized as susceptible by microdilution, were detected as non-susceptible by MBT ASTRA. A categorical agreement of $82 \%$ was obtained between the two methods, and sensitivity and specificity of $100 \%$ and $73 \%$ were calculated, respectively (Table 4) (Supplementary Table 1).

\section{MBT ASTRA on Positive Blood Culture}

The applicability of the MBT ASTRA for detecting yeast strains non-susceptible to echinocandins was directly investigated for cells derived from seeded blood cultures. Initially, spectra were analyzed by the MBT ASTRA prototype to determine the breakpoints and thresholds for the respective three antifungals. Afterwards, 14 susceptible and 6 non-susceptible strains (against anidulafungin and micafungin) were tested at the respective

TABLE 2 | In vitro echinocandin class drugs susceptibility test using CLSI microdilution and MBT ASTRA for 50 C. auris isolates.

\begin{tabular}{lccr}
\hline Methods & \multicolumn{3}{c}{ Total no (Susceptible/Resistant) } \\
\cline { 2 - 4 } & Anidulafungin & Micafungin & Caspofungin \\
\hline CLSI & $50(44 / 6)$ & $50(44 / 6)$ & $50(33 / 17)$ \\
MBT ASTRA & $50(43 / 7)$ & $50(42 / 8)$ & $50(24 / 26)$
\end{tabular}

TABLE 1 | Identification of 50 C. auris isolates derived from SDA plate agar and positive blood cultures by MALDI-TOF MS, respectively.

\begin{tabular}{|c|c|c|c|c|c|c|}
\hline \multirow[t]{2}{*}{ Strains/culture medium } & \multirow{2}{*}{$\begin{array}{l}\text { Number of } \\
\text { strains }\end{array}$} & \multicolumn{3}{|c|}{ No of log (score) values } & \multirow{2}{*}{$\begin{array}{c}\text { No of correct identified } \\
\text { samples (\%) }\end{array}$} & \multirow[t]{2}{*}{ Accuracy } \\
\hline & & $\geq 2$ & $<2$ and $\geq 1.7$ & $\leq 1.7$ & & \\
\hline C. auris/Plate agar (SDA) & 50 & 47 & 3 & - & $50(100)$ & $100 \%$ \\
\hline C. auris/positive blood & 50 & 46 & 4 & - & $50(100)$ & $100 \%$ \\
\hline
\end{tabular}

culture (BC) 
TABLE 3 | Comparison of suggested breakpoints against echinocandin class drugs for C. auris tested by CLSI microdilution and MBT ASTRA.

\begin{tabular}{|c|c|c|c|}
\hline \multirow[t]{2}{*}{ Methods } & \multicolumn{3}{|c|}{$\mathrm{MIC} \mu \mathrm{g} / \mathrm{ml}$} \\
\hline & Anidulafungin & Micafungin & Caspofungin \\
\hline CLSI & $\mathrm{R}>4 \mu \mathrm{g} / \mathrm{ml} \quad \mathrm{S} \leq 4 \mu \mathrm{g} / \mathrm{ml}$ & $\mathrm{R}>8 \mu \mathrm{g} / \mathrm{ml} \mathrm{S} \leq 8 \mu \mathrm{g} / \mathrm{ml}$ & $\mathrm{R} \geq 1 \mu \mathrm{g} / \mathrm{ml} \mathrm{S}<1 \mu \mathrm{g} / \mathrm{ml}$ \\
\hline MBT ASTRA (SDA) & $\mathrm{R}>4 \mu \mathrm{g} / \mathrm{ml} \mathrm{S} \leq 4 \mu \mathrm{g} / \mathrm{ml}$ & $\mathrm{R}>8 \mu \mathrm{g} / \mathrm{ml} \mathrm{S} \leq 8 \mu \mathrm{g} / \mathrm{ml}$ & $\mathrm{R} \geq 2 \mu \mathrm{g} / \mathrm{ml} \mathrm{S}<2 \mu \mathrm{g} / \mathrm{ml}$ \\
\hline MBT ASTRA (blood culture bottles) & $\mathrm{R}>1 \mu \mathrm{g} / \mathrm{ml} \mathrm{S} \leq 1 \mu \mathrm{g} / \mathrm{ml}$ & $\mathrm{R}>4 \mu \mathrm{g} / \mathrm{ml} \mathrm{S} \leq 4 \mu \mathrm{g} / \mathrm{ml}$ & $\mathrm{R} \geq 1 \mu \mathrm{g} / \mathrm{ml} \mathrm{S}<1 \mu \mathrm{g} / \mathrm{ml}$ \\
\hline
\end{tabular}

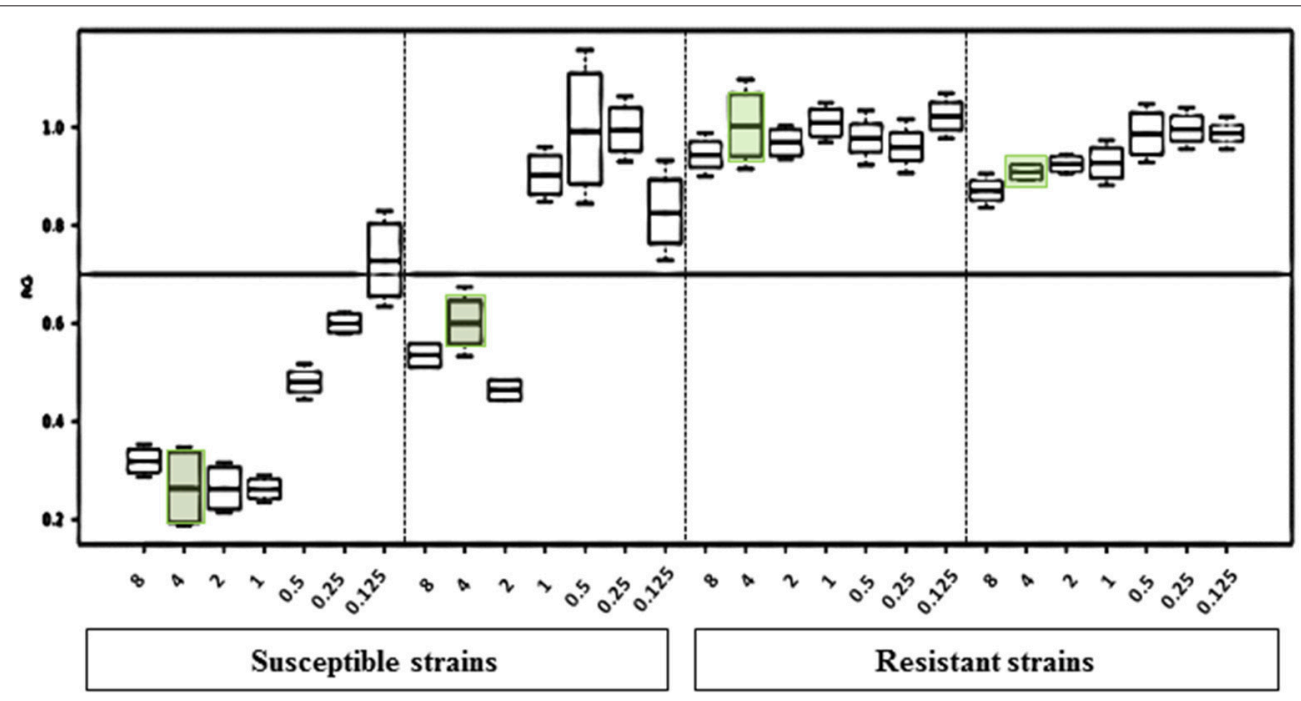

FIGURE 1 | Results of the MBT ASTRA prototype software evaluation. The relative growth values of 2-fold serial dilutions of anidulafungin of 2 susceptible and 2 resistant $C$. auris strains after $6 \mathrm{~h}$ incubation show distinct differences. The susceptibility/resistance threshold was set at a RG value of 0.7 . For susceptible strains, at the concentration of $4 \mu \mathrm{g} / \mathrm{ml}$ anidulafungin a significant reduction of the relative growth was observed.

breakpoints and thresholds. The results for anidulafungin showed that the breakpoint was decreased to $1 \mu \mathrm{g} / \mathrm{mL}$ at a threshold of 0.7, compared to the results of cells cultured on SDA (Table 3). Beside this result, the breakpoint was reduced to $4 \mu \mathrm{g} / \mathrm{mL}$ for micafungin when an RG threshold of 0.7 was applied (Table 3). All 6 non-susceptible and 14 susceptible strains were correctly separated at these breakpoints of the corresponding antifungals and thresholds (Figure 3). For caspofungin, 6 out of 7 non-susceptible strains and twelve out of thirteen susceptible strains were correctly detected. In this case, the 2 strains that were misidentified, had been correctly detected by MBT ASTRA from colonies grown on SDA. These data demonstrate that the breakpoint for caspofungin has to be decreased to $1 \mu \mathrm{g} / \mathrm{mL}$ at a threshold of 0.7 (Table 3) when using MBT ASTRA.

\section{DISCUSSION}

This study investigated the identification of 50 C. auris isolates by MALDI-TOF MS, suggested CLSI microdilution cut-offs and evaluated MBT ASTRA for AFST on echinocandin. The results from this study demonstrate that a rapid MALDITOF MS technology not only can be used to unequivocally identify $C$. auris, but it has the potential to be successfully applied in rapid AFST. Recently, attention has focused on the identification of $C$. auris to distinguish it from close relatives like C. haemulonii using diverse biochemical-based testing, molecular, and commercial methods. A total of $50 \mathrm{C}$. auris isolates were correctly identified by MALDI-TOF MS with an accuracy of $100 \%$ when cultured on SDA and isolated from positive blood cultures. This was for a first time that applicability of MALDI-TOF MS was used to identify C. auris derived from positive blood cultures. This finding broadly supports the work of other studies in this area, demonstrating the high accuracy of MALDI-TOF MS for the identification of C. auris based on a well-established reference database, compared to other available methods. For instance, the results of this study are in agreement with those obtained by Bao JR et al., who more recently showed a high performance of the MALDI-TOF MS in identifying 23 clinical isolates of $C$. auris that were cultured on three different media. Their study indicated that the highest log scores were achieved for isolates grown on SDA (Bao et al., 2018).

Additionally, the present study demonstrates the successful applicability of MBT ASTRA for the detection of non-susceptible C. auris isolates to echinocandin drugs cultured on SDA. The most interesting finding was that 49 and 48 out of fifty C. auris isolates were correctly labeled as either susceptible or non-susceptible to anidulafungin and micafungin by MBT 


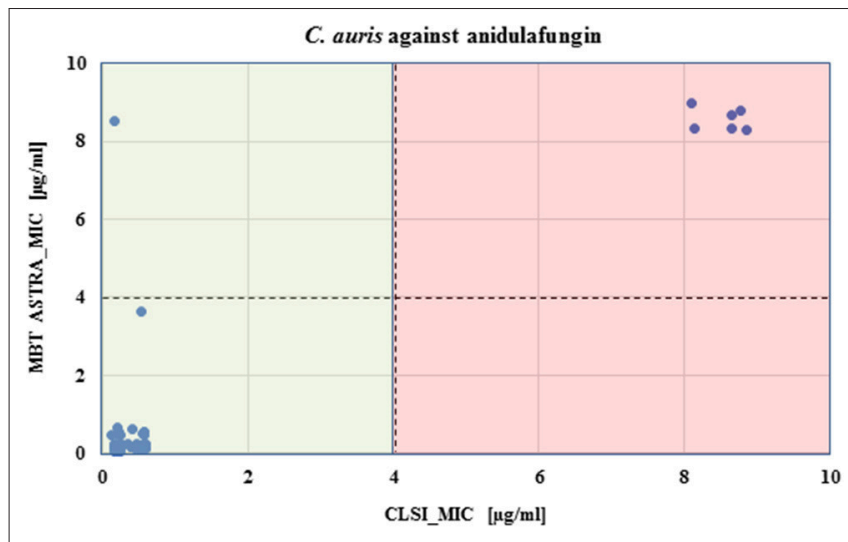

FIGURE 2 | Comparison of CLSI microdilution MICs and MBT ASTRA MICs for $C$. auris isolates $(n=50)$ against anidulafungin. For each isolate, the MIC value derived by MBT ASTRA ( $y$ axis) was plotted against the MIC obtained by microdilution ( $x$ axis) for anidulafungin. The colored boxes indicate the MIC ranges according to CLSI; green, susceptible and red, resistant. The horizontal dashed line indicates the suggested cutoff defined for MBT ASTRA MIC, and the vertical dashed line shows the suggested cutoff determined for CLSI. A high agreement was observed between both approaches, and only one isolate susceptible against anidulafungin was wrongly detected by MBT ASTRA.

TABLE 4 | Sensitivity, specificity, and categorical agreement of MBT ASTRA in comparison to CLSI microdilution results for anidulafungin, micafungin, and caspofungin.

\begin{tabular}{|c|c|c|c|}
\hline MBT ASTRA & Anidulafungin (\%) & Micafungin (\%) & Caspofungin (\%) \\
\hline Sensitivity & 100 & 100 & 100 \\
\hline Specificity & 98 & 95.5 & 73 \\
\hline Categorical agreement & 98 & 96 & 82 \\
\hline
\end{tabular}

ASTRA, respectively. A sensitivity of $100 \%$ indicated that all 6 isolates non-susceptible against the respective antifungals were successfully detected. Notably, the same susceptible isolate was mis-identified as resistant for both antifungals even after repetition. The reason for this observation could be that this strain revealed an MIC in the microdilution that is next the breakpoint concentration. Considering that this strain had not been sequenced together with the allowed variance of the microdilution, it could be that this strain is indeed resistant. Further analysis is required to clarify this result.

To achieve valid results by MBT ASTRA, it is essential to first check the growth in the control setups. This was done by introducing a threshold for the minimum required growth calculated by the prototype software. Those strains with sufficient growth in the control setups were considered for further evaluation of sensitivity and specificity of the MBT ASTRA. Taken together, categorical agreement between MBT ASTRA and the CLSI was very high; $98 \%$ and $96 \%$ for anidulafungin and micafungin, respectively. Moreover, in the present study, the cut-offs found by the CLSI reference method for $C$. auris anidulafungin susceptibility testing confirmed the results obtained by other studies (Arendrup et al., 2017b; Chowdhary et al., 2018; Kordalewska et al., 2018; Spivak and

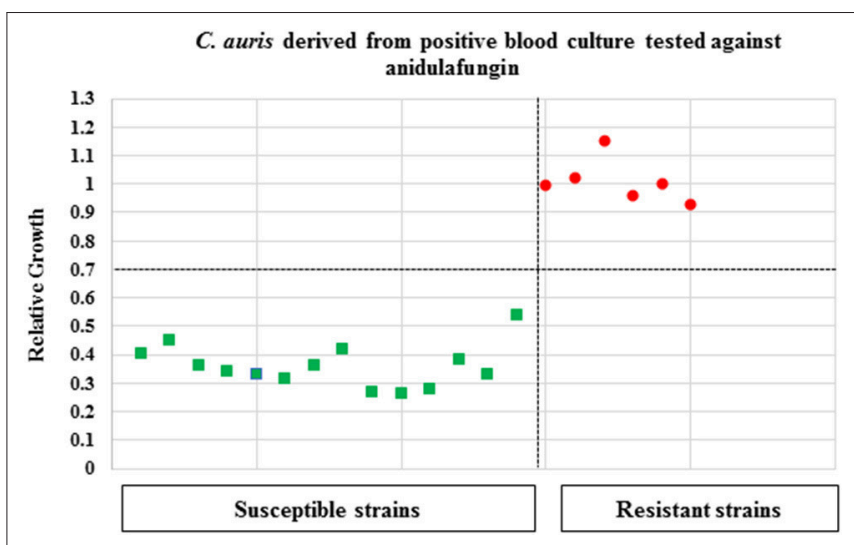

FIGURE 3 | MBT ASTR4 of $C$. auris isolates derived from positive blood cultures. A total of $20 \mathrm{C}$. auris isolates (resistant $=6$, susceptible $=14$ ) derived from positive blood cultures were tested against anidulafungin. The MBT ASTRA breakpoint was determined at I $\mu \mathrm{g} / \mathrm{ml}$ and an RG threshold of 0.7 for anidulafungin. All resistant and susceptible isolates were correctly detected.

Hanson, 2018), and the breakpoint found by MBT ASTRA was identical to the suggested (CDC) CLSI method. Importantly, the same breakpoint was obtained much more rapidly by MBT ASTRA, which requires up to $6 \mathrm{~h}$, compared to $24 / 48 \mathrm{~h}$ used for the CLSI method in this study. For micafungin, the suggested cut-offs for the CLSI microdilution and MBT ASTRA were also identical, but these cut-offs were higher compared to those of other studies (Arendrup et al., 2017b; Chowdhary et al., 2018; Kordalewska et al., 2018). A recent study reported that micafungin to be the most potent echinocandin with an MIC approximately equal to that of anidulafungin (Kordalewska et al., 2018). In this study, the higher MICs obtained by the CLSI method and MBT ASTRA might be an epidemiological impact on fungal infections with a shift toward a reduced susceptibility of the strains against echinocandins (Arendrup and Patterson, 2017; Lockhart et al., 2017). Notably, results with caspofungin had lower specificity and sensitivity than anidulafungin or micafungin, with a categorical agreement between the CLSI microdilution and MBT ASTRA of $82 \%$. Furthermore, the breakpoint found by MBT ASTRA was one serial dilution higher than that applied for the CLSI method. In accordance with these results, previous study reported challenges in identification of FKS1 WT C. auris isolates for caspofungin antifungal susceptibility testing due to the presence of an 'Eagle effect' (Vanstraelen et al., 2013). This phenomenon has been notably reported for the caspofungin-caused reduction of the antifungal activity at high concentrations (Vanstraelen et al., 2013). As mentioned in the study by Espinel- Ingroff et al., variability of caspofungin MICs has been observed for Candida spp. used CLSI and EUCAST methods from different clinical laboratories. They have reported that most of the wild-type (WT) isolates (e.g., C. glabrata and C. krusei) were detected as either non-WT or resistant isolates (Espinel-Ingroff et al., 2013). Accordingly, Kordalewska et al. (2018) examined AFST of caspofungin for 106 C. auris isolates and found that only isolates with mutations in FKS1 were resistant to echinocandins. Therefore, 
it is recommended that routine microdilution methods be avoided when detecting resistant strains without gene sequencing (Kordalewska et al., 2018). However, the reduced susceptibility of C. auris isolates to one or more echinocandin class drugs has been reported in several studies (Arendrup et al., 2017b; Kordalewska et al., 2018). A further extension of this MBT ASTRA investigation was to analyse its performance on positive blood culture samples. For anidulafungin and micafungin, all susceptible and non-susceptible strains were labeled correctly, with complete agreement between the CLSI method and MBT ASTRA. The breakpoint concentration was decreased by two and one serial dilution(s) for anidulafungin and micafungin, respectively. For caspofungin, one susceptible and one nonsusceptible strain were mis-labeled resulting in a categorical agreement of $90 \%$. Thus, for caspofungin, these results from blood culture bottles were more in agreement with CLSI method than those obtained from SDA.

Findings reported here shed new light on rapid and accurate antifungal susceptibility testing for echinocandin by MALDITOF MS. A new performance of MALDI-TOF MS for rapid AFST based on changes in the profile spectra in the presence of antifungals was recently introduced by Vella et al. (2013), but however, they revealed that this approach could not perfectly be applied for C. glabrata strains resistant to anidulafungin with known FKS2 mutations (Vella et al., 2017). In this study, the CLSI method was considered as a gold standard, however, it is not a perfect method and some susceptible isolates are not clearly detected by the CLSI method. Hence, the MBT ASTRA might be even more helpful in the future. In summary, MBT ASTRA has been shown to be applicable for rapid AFST in C. albicans, C. glabrata (Vatanshenassan et al., 2018), and C. auris. Applicability for further yeast species and antifungals will be necessary.

\section{ETHICS STATEMENT}

According to common sense, for this study no ethical approval from an ethics committee is required, because all strains were derived either from strain collections or stock cultures of routine samples. The isolates used in this non-clinical in vitro study

\section{REFERENCES}

Arendrup, M. C., Meletiadis, J., Mouton, J. W., Lagrou, K., Hamal, P., Guinea, J., et al. (2017a). Method for the determination of broth dilution minimum inhibitory concentrations of antifungal agents for yeasts. EUCAST E.DEF 7.3.1, $1-21$.

Arendrup, M. C., and Patterson, T. F. (2017). Multidrug-resistant candida: epidemiology, molecular mechanisms, and treatment. J. Infect. Dis. 216(Suppl. 3), S445-S451. doi: 10.1093/infdis/jix131

Arendrup, M. C., Prakash, A., Meletiadis, J., Sharma, C., and Chowdhary, A. (2017b). Comparison of EUCAST and CLSI reference microdilution MICs of eight antifungal compounds for Candida auris and associated tentative epidemiological cutoff values. Antimicrob. Agents Chemother. 61, e00485e00417. doi: 10.1128/AAC.00485-17

Bao, J. R., Master, R. N., Azad, K. N., Schwab, D. A., Clark, R. B., Jones, R. S., et al. (2018). Rapid, accurate identification of Candida auris by using a novel matrix-assisted laser desorption ionization-time of flight mass spectrometry were obtained during routine patient care for which no written informed consent is required. The local Institutional Review Board of the hospital determined that ethics approval and consent from admitted patients was not required according to national and institutional guidelines.

\section{AUTHOR CONTRIBUTIONS}

MV contributed to the conceptualization, the investigation, the methodology, and the writing of the original draft. MK contributed to the conceptualization the methodology, the scientific advice, the project administration, the review, and editing of the manuscript, and the funding acquisition. KS contributed to the conceptualization, the methodology, the scientific advice, the project administration, and the review and editing of the manuscript. TB reviewed and edited the manuscript. JM reviewed and edited the manuscript, contributed to providing a sample, and scientific advice. JB and AC reviewed and edited the manuscript, and provided a sample. RB-A provided a sample.

\section{FUNDING}

This work was funded by the European Union (OPATHY, Project ID: 642095; Program H2020-EU.1.3.1). MK, MV, and TB are nominated under this grant.

\section{ACKNOWLEDGMENTS}

We are grateful to Centers for Disease Control and Prevention (CDC) for providing some of the strains used in this study.

\section{SUPPLEMENTARY MATERIAL}

The Supplementary Material for this article can be found online at: https://www.frontiersin.org/articles/10.3389/fcimb. 2019.00020/full\#supplementary-material ${ }^{1}$

\footnotetext{
${ }^{1}$ These strains were identified by MALDI-TOF MS.
}

(MALDI-TOF MS) database (Library). J. Clin. Microbiol. 56, e01700-17. doi: 10.1128/JCM.01700-1741

Ben-Ami, R., Berman, J., Novikov, A., Bash, E., Shachor-Meyouhas, Y., Zakin, S., et al. (2017). Multidrug-resistant candida haemulonii and C. auris, Tel Aviv, Israel. Emerg. Infect. Dis. 23, 195-203. doi: 10.3201/eid2302.161486

Cendejas-Bueno, E., Kolecka, A., Alastruey-Izquierdo, A., Theelen, B., Groenewald, M., Kostrzewa, M., et al. (2012). Reclassification of the Candida haemulonii complex as Candida haemulonii (C. haemulonii group I), C. duobushaemulonii sp. nov. (C. haemulonii group II), and $C$. haemulonii var. vulnera var. nov.: three multiresistant human pathogenic yeasts. J. Clin. Microbiol. 50, 3641-3651. doi: 10.1128/JCM. 02248-12

Ceyssens, P. J., Soetaert, K., Timke, M., Van de Bossche, A., Sparbier, K., De Cremer, K., et al. (2017). Matrix-assisted laser desorption ionizationtime of flight mass spectrometry for combined species identification and drug sensitivity testing in Mycobacteria. J. Clin. Microbiol. 55, 624-634. doi: 10.1128/JCM.02089-16 
Chowdhary, A., Prakash, A., Sharma, C., Kordalewska, M., Kumar, A., Sarma, S., et al. (2018). A multicentre study of antifungal susceptibility patterns among 350 Candida auris isolates (2009-17) in india: role of the ERG11 and FKS1 genes in azole and echinocandin resistance. J. Antimicrob. Chemother. 73, 891-899. doi: $10.1093 / \mathrm{jac} / \mathrm{dkx} 480$

Chowdhary, A., Sharma, C., Duggal, S., Agarwal, K., Prakash, A., Singh, P. K., et al. (2013). New clonal strain of Candida auris, Delhi, India. Emerg. Infect. Dis. 19, 1670-1673. doi: 10.3201/eid1910.130393

Chowdhary, A., Sharma, C., and Meis, J. F. (2017). Candida auris: a rapidly emerging cause of hospital-acquired multidrug-resistant fungal infections globally. PLoS Pathog. 13:e1006290. doi: 10.1371/journal.ppat. 1006290

Chowdhary, A., Voss, A., and Meis, J. F. (2016). Multidrug-resistant Candida auris: "new kid on the block" in hospital-associated infections? J. Hosp. Infect. 94, 209-212. doi: 10.1016/j.jhin.2016.08.004

Clark, A. E., Kaleta, E. J., Arora, A., and Wolk, D. M. (2013). Matrix-assisted laser desorption ionization-time of flight mass spectrometry: a fundamental shift in the routine practice of clinical microbiology. Clin. Microbiol. Rev. 26, 547-603. doi: 10.1128/CMR.00072-12

Clinical and Laboratory Standards Institute. Performance Standards for Antifungal Susceptibility Testing of Yeasts; approved, standard. (2017). CLSI document M60, 1st Edn. Wayne, PA: Clinical and Laboratory Standards Institute, 26.

Cortegiani, A., Misseri, G., Fasciana, T., Giammanco, A., Giarratano, A., and Chowdhary, A. (2018). Epidemiology, clinical characteristics, resistance, and treatment of infections by Candida auris. J. Inten. Care 6:69. doi: 10.1186/s40560-018-0342-4

Croxatto, A., Prod'hom, G., and Greub, G. (2012). Applications of MALDI-TOF mass spectrometry in clinical diagnostic microbiology. FEMS Microbiol. Rev. 36, 380-407. doi: 10.1111/j.1574-6976.2011. 00298.x

Espinel-Ingroff, A., Arendrup, M. C., Pfaller, M. A., Bonfietti, L. X., Bustamante, B., Canton, E., et al. (2013). Interlaboratory variability of Caspofungin MICs for Candida spp. Using CLSI and EUCAST methods: should the clinical laboratory be testing this agent? Antimicrob. Agents Chemother. 57, 5836-5842. doi: 10.1128/AAC.01519-13

Ghosh, A. K., Paul, S., Sood, P., Rudramurthy, S. M., Rajbanshi, A., Jillwin, T. J., et al. (2015). Matrix-assisted laser desorption ionization time-of-flight mass spectrometry for the rapid identification of yeasts causing bloodstream infections. Clin. Microbiol. Infect. 21, 372-378. doi: 10.1016/j.cmi.2014. 11.009

Gibb, S., and Strimmer, K. (2012). MALDIquant: a versatile r package for the analysis of mass spectrometry data. Bioinforma Oxf. Engl. 28, 2270-2271. doi: 10.1093/bioinformatics/bts447

Girard, V., Mailler, S., Chetry, M., Vidal, C., Durand, G., van Belkum, A., et al. (2016). Identification and typing of the emerging pathogen Candida auris by matrix-assisted laser desorption ionisation time of flight mass spectrometry. Mycoses 59, 535-538. doi: 10.1111/myc.12519

Jeffery-Smith, A., Taori, S. K., Schelenz, S., Jeffery, K., Johnson, E. M., Borman, A., et al. (2018). Candida auris: a review of the literature. Clin. Microbiol. Rev. 3, e00029-e00017. doi: 10.1128/CMR.00029-17

Kathuria, S., Singh, P. K., Sharma, C., Prakash, A., Masih, A., Kumar, A., et al. (2015). Multidrug-resistant Candida auris misidentified as Candida haemulonii: characterization by matrix-assisted laser desorption ionizationtime of flight mass spectrometry and dna sequencing and its antifungal susceptibility profile variability by vitek 2, CLSI broth microdilution, and etest method. J. Clin. Microbiol. 53, 1823-1830. doi: 10.1128/JCM. 00367-15

Khan, Z., Ahmad, S., Al-Sweih, N., Joseph, L., Alfouzan, W., and Asadzadeh, M. (2018). Increasing prevalence, molecular characterization and antifungal drug susceptibility of serial Candida auris isolates in Kuwait. PLoS ONE 13:e0195743. doi: 10.1371/journal.pone.0195743

Kolecka, A., Sanguinetti, M., Boekhout, T., and Posteraro B. (2016). "Identification, typing and susceptibility testing of fungi (incl. Yeasts) by MALDI-TOF MS," in MALDI-TOF Mass Spectrometry in Microbiology, eds M. Kostrzewa and S. Schubert (Norfolk, UK: British Library Cataloguing-in-Publication Data, Caister Academic Press), 49-78.

Kordalewska, M., Lee, A., Park, S., Berrio, I., Chowdhary, A., Zhao, Y., et al. (2018). Understanding echinocandin resistance in the emerging pathogen Candida auris. Antimicrob. Agents Chemother. 62, e00238-18. doi: 10.1128/AAC.00238-18.

Lange, C., Schubert, S., Jung, J., Kostrzewa, M., and Sparbier, K. (2014). Quantitative matrix-assisted laser desorption ionization-time of flight mass spectrometry for rapid resistance detection. J. Clin. Microbiol. 52, 4155-4162. doi: 10.1128/JCM.01872-14

Lee, W. G., Shin, J. H., Uh, Y., Kang, M. G., Kim, S. H., Park, K. H., et al. (2011). First three reported cases of nosocomial fungemia caused by Candida auris. J. Clin. Microbiol. 49, 3139-3142. doi: 10.1128/JCM. 00319-11

Lockhart, S. R., Etienne, K. A., Vallabhaneni, S., Farooqi, J., Chowdhary, A., Govender, N. P., et al. (2017). Simultaneous emergence of multidrug-resistant Candida auris on 3 continents confirmed by whole-genome sequencing and epidemiological analyses. Clin. Infect. Dis. 64, 134-140. doi: 10.1093/cid/ ciw691

Mathur, P., Hasan, F., Singh, P. K., Malhotra, R., Walia, K., and Chowdhary, A. (2018). Five-year profile of candidaemia at an indian trauma centre: high rates of Candida auris blood stream infections. Mycoses 61, 674-680. doi: 10.1111/myc. 12790

Meis, J. F., and Chowdhary, A. (2018). Candida auris: a global fungal public health threat. Lancet Infect. Dis. 18, 1298-1299. doi: 10.1016/S1473-3099(18) 30609-1

Prakash, A., Sharma, C., Singh, A., Kumar Singh, P., Kumar, A., Hagen, F., et al. (2016). Evidence of genotypic diversity among Candida auris isolates by multilocus sequence typing, matrix-assisted laser desorption ionization time-of-flight mass spectrometry and amplified fragment length polymorphism. Clin. Microbiol. Infect. 22, 277.e1-9. doi: 10.1016/j.cmi.2015. 10.022

Ruiz-Gaitán, A., Moret, A. M., Tasias-Pitarch, M., Aleixandre-López, A. I., Martínez-Morel, H., Calabuig, E., et al. (2018). An outbreak due to Candida auris with prolonged colonisation and candidaemia in a tertiary care european hospital. Mycoses 61, 498-505. doi: 10.1111/myc. 12781

Saris, K., Meis, J. F., and Voss, A. (2018). Candida auris. Curr. Opin. Infect. Dis. 31, 334-340. doi: 10.1097/QCO.0000000000000469

Satoh, K., Makimura, K., Hasumi, Y., Nishiyama, Y., Uchida, K., and Yamaguchi, H. (2009). Candida auris sp. nov., a novel ascomycetous yeast isolated from the external ear canal of an inpatient in a Japanese hospital. Microbiol. Immunol. 53, 41-44. doi: 10.1111/j.1348-0421.2008. 00083.x

Sauer, S., Freiwald, A., Maier, T., Kube, M., Reinhardt, R., Kostrzewa, M., et al. (2008). Classification and identification of bacteria by mass spectrometry and computational analysis. PLoS ONE 3:e2843. doi: 10.1371/journal.pone. 0002843

Schelenz, S., Hagen, F., Rhodes, J. L., Abdolrasouli, A., Chowdhary, A., Hall, A., et al. (2016). First hospital outbreak of the globally emerging Candida auris in a European hospital. Antimicrob. Resist. Infect. Control 5:35. doi: 10.1186/s13756-016-0132-5.

Sears, D., and Schwartz, B. S. (2017). Candida auris: an emerging multidrugresistant pathogen. Int. J. Infect. Dis. 63, 95-98. doi: 10.1016/j.ijid.2017.08.017

Shin, J. H., Kim, M. N., Jang, S. J., Ju, M. Y., Kim, S. H., Shin, M. G., et al. (2012). Detection of amphotericin b resistance in Candida haemulonii and closely related species by use of the etest, vitek-2 yeast susceptibility system, and CLSI and EUCAST broth microdilution methods. J. Clin. Microbiol. 50, 1852-1855. doi: 10.1128/JCM.06440-11

Spivak, E. S., and Hanson, K. E. (2018). Candida auris: an emerging fungal pathogen. J. Clin. Microbiol. 56, e01588-17. doi: 10.1128/JCM. 01588-17.19

Tsay, S., Kallen, A., Jackson, B. R., Chiller, T. M., and Vallabhaneni, S. (2018). Approach to the investigation and management of patients with Candida auris, an emerging multidrug-resistant yeast. Clin. Infect. Dis. 66, 306-311. doi: 10.1093/cid/cix744

Vanstraelen, K., Lagrou, K., Maertens, J., Wauters, J., Willems, L., and Spriet, I. (2013). The eagle-like effect of echinocandins: what's in a name? Exp. Rev. Anti. Infect. Ther. 11, 1179-1191. doi: 10.1586/14787210.2013. 841543

Vatanshenassan, M., Boekhout, T., Lass-Flörl, C., Lackner, M., Schubert, S., Kostrzewa, M., et al. (2018). MBT ASTRA: proof-of-concept for a 
rapid MALDI-TOF MS based method to detect caspofungin resistance in Candida albicans and Candida glabrata. J. Clin. Microbiol. 56, 9. doi: 10.1128/JCM.00420-18

Vella, A., De Carolis, E., Mello, E., Perlin, D. S., Sanglard, D., Sanguinetti, M., et al. (2017). Potential use of MALDI-ToF mass spectrometry for rapid detection of antifungal resistance in the human pathogen Candida glabrata. Sci. Rep. 7, 9099. doi: 10.1038/s41598-017-09329-4

Vella, A., De Carolis, E., Vaccaro, L., Posteraro, P., Perlin, D. S., Kostrzewa, M., et al. (2013). Rapid antifungal susceptibility testing by matrix-assisted laser desorption ionization-time of flight mass spectrometry analysis. J. Clin. Microbiol. 51, 2964-2969. doi: 10.1128/JCM.00903-13

Vincent, J. L., Rello, J., Marshall, J., Silva, E., Anzueto, A., Martin, C. D., et al. (2009). International study of the prevalence and outcomes of infection in intensive care units. JAMA 302, 2323-2329. doi: 10.1001/jama. 2009.1754

Vlek, A., Kolecka, A., Khayhan, K., Theelen, B., Groenewald, M., Boel, E., et al. (2014). Interlaboratory comparison of sample preparation methods, database expansions, and cutoff values for identification of yeasts bymatrix- assisted laser desorption ionization-time of flight mass spectrometry using a yeast test panel. J. Clin. Microbiol. 52, 3023-3029. doi: 10.1128/JCM. 00563-14

Conflict of Interest Statement: MK, KS, and MV are employees of the mass spectrometry company Bruker Daltonik GmbH.

The remaining authors declare that the research was conducted in the absence of any commercial or financial relationships that could be construed as a potential conflict of interest.

Copyright (C) 2019 Vatanshenassan, Boekhout, Meis, Berman, Chowdhary, Ben-Ami, Sparbier and Kostrzewa. This is an open-access article distributed under the terms of the Creative Commons Attribution License (CC BY). The use, distribution or reproduction in other forums is permitted, provided the original author(s) and the copyright owner(s) are credited and that the original publication in this journal is cited, in accordance with accepted academic practice. No use, distribution or reproduction is permitted which does not comply with these terms. 